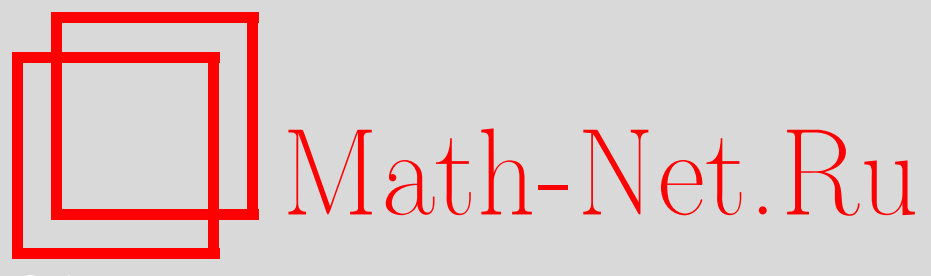

Д. В. Костин, Применение формулы Маслова для асимптотического решения одной задачи об упругих деформациях, Матем. заметки, 2008, том 83, выпуск 1, 50-60

DOI: https://doi.org/10.4213/mzm4335

Использование Общероссийского математического портала Math-Net.Ru подразумевает, что вы прочитали и согласны с пользовательским соглашением http://www . mathnet.ru/rus/agreement

Параметры загрузки:

IP : 54.89 .56 .158

26 апреля 2023 г., 13:13:05

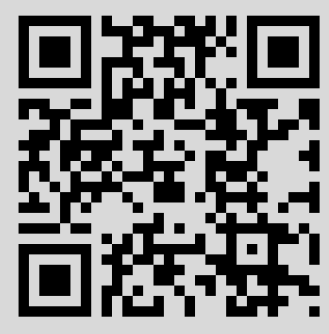


Том 83 выпуск 1 январь 2008

УДК 517.9

\section{Применение формулы Маслова для асимптотического решения одной задачи об упругих деформациях}

\section{Д. В. Костин}

Предложена схема бифуркационного анализа равновесных конфигураций слабо неоднородной упругой балки на упругом основании в условиях двумодового вырождения, обобщающая схему Даринского-Сапронова, ранее разработанную для случая однородной балки. Переход к неоднородной балке потребовал замены условия постоянства пары собственных векторов оператора $\mathscr{A}$ из линейной части уравнения (в нуле) на условие существования пары векторов, гладко зависящих от параметров, линейная оболочка которых инвариантна относительно $\mathscr{A}$. Показано, что такой пары достаточно для построения главной части ключевой функции и для проведения анализа ветвлений равновесных конфигураций балки. Построение искомой пары векторов основано на формуле ортогонального проектора на корневое подпространство $\mathscr{A}$ (из теории возмущений самосопряженных операторов по версии Маслова). Проанализировано влияние характера неоднородности балки на форму ее прогиба.

Библиография: 11 названий.

В настоящей статье предложена схема анализа бифуркаций равновесных конфигураций слабо неоднородной упругой балки на упругом основании в условиях двумодового вырождения. Решение аналогичной задачи в случае однородной балки ранее было дано Даринским и Сапроновым [1], [2]. Переход к случаю неоднородной балки потребовал перестройки в исследовательской схеме Даринского и Сапронова, в основе которой лежало условие постоянства пары собственных функций $e_{1}, e_{2}$ второго дифференциала (в нуле) функционала энергии. В случае неоднородной балки это условие нарушается и, более того, оно не допускает прямого обобщения, например, в виде условия существования непрерывного семейства собственных функций. В предлагаемой схеме условие постоянных собственных функций заменено условием существования пары гладких векторных полей $\widetilde{e}_{1}, \widetilde{e}_{2}$, линейная оболочка которых инвариантна относительно второго дифференциала в нуле. Наличие такой пары достаточно для построения главной части ключевой функции и, как следствие, для проведения анализа ветвления равновесных конфигураций балки.

В построении требуемой пары векторных полей главную роль сыграла взятая из монографии Маслова [3] формула ортогонального проектора (на линейную оболочку $\left.\widetilde{e}_{1}, \widetilde{e}_{2}\right)$. 
Рассмотрение случая слабо неоднородной балки приводит к “понижению" симметрии ключевой функции и увеличению на единицу размерности ее базы деформации (появляется дополнительное квадратичное слагаемое в главной части ключевой функции). Анализ бифуркационных диаграмм и бифурцирующих критических точек в этой ситуации несколько усложняется [4], но выявляет новые бифуркационные эффекты.

Случай однородной балки. Колебания и волновые движения упругой балки на упругом основании изучали Митропольский, Мосеенков [5], Томпсон, Стюарт [6], Бардин, Фурта [7] и др. Простейшая нелинейная (промасштабированная) модель движений однородной балки описывается уравнением

$$
\frac{\partial^{2} w}{\partial t^{2}}+\frac{\partial^{4} w}{\partial x^{4}}+\kappa \frac{\partial^{2} w}{\partial x^{2}}+\alpha w+w^{3}=0,
$$

где $w$ - прогиб балки (поле смещений точек средней линии упругой балки, расположенной вдоль оси $x$ ). Заметим, что аналогичное уравнение возникает и в теории кристаллов [8], в которой $w$ - параметр порядка.

Как известно, первый шаг в изучении такой задачи - отыскание равновесных (стационарных) состояний, определяемых уравнением

$$
\frac{d^{4} w}{d x^{4}}+\kappa \frac{d^{2} w}{d x^{2}}+\alpha w+w^{3}=0
$$

Если рассмотреть стандартные краевые условия

$$
w(0)=w(1)=w^{\prime \prime}(0)=w^{\prime \prime}(1)=0,
$$

то полученная граничная задача может допускать 2-мерные вырождения, порождающие 2-модовые бифуркации с интересными геометрическими и физическими эффектами. Решение этой задачи проводится на основе конечномерной редукции [9] к описанию экстремалей 2-параметрического семейства полиномов от двух переменных. В случае слабо неоднородной балки также допускается аналогичное сведение, но при этом в полиноме появляется дополнительное слагаемое, создающее более сложную картину бифуркационного поведения решений.

Уравнение (1) является уравнением Эйлера для экстремалей функционала

$$
V=\int_{0}^{1}\left(\frac{1}{2}\left(\left(\frac{d^{2} w}{d x^{2}}\right)^{2}-\kappa\left(\frac{d w}{d x}\right)^{2}+\alpha w^{2}\right)+\frac{w^{4}}{4}\right) d x .
$$

Двумерное вырождение нулевой экстремали происходит при

$$
\kappa=\kappa_{1}:=\left(p^{2}+q^{2}\right) \pi^{2}, \quad \alpha=\alpha_{1}:=p^{2} q^{2} \pi^{4}, \quad p, q \in \mathbb{N},
$$

со стандартным базисом (модами бифуркации) ядра второго дифференциала

$$
e_{1}=\sqrt{2} \sin (p \pi x), \quad e_{2}=\sqrt{2} \sin (q \pi x) .
$$

Ниже предполагается, что $p=1, q=2$ и, соответственно, $\kappa_{1}=5 \pi^{2}, \alpha_{1}=4 \pi^{4}$ (эти значения являются наименьшими из тех, при которых происходит 2-мерное вырождение; в остальных случаях анализ аналогичен). 
При сделанных предположениях анализ возможных бифуркационных эффектов осуществляется посредством редукции Ляпунова-Шмидта [2] к (ключевой) функции (двух переменных)

$$
\begin{gathered}
W(\xi, \delta)=\inf _{w:\left\langle w, e_{1}\right\rangle=\xi_{1},\left\langle w, e_{2}\right\rangle=\xi_{2}} V\left(w, \alpha_{1}+\delta_{1}, \kappa_{1}+\delta_{2}\right), \\
\xi=\left(\xi_{1}, \xi_{2}\right), \quad \delta=\left(\delta_{1}, \delta_{2}\right) .
\end{gathered}
$$

Функционал (3) инвариантен относительно инволюций $J_{1}, J_{2}$ :

$$
J_{2}(p)(x):=p(1-x), \quad J_{1}:=-J_{2} .
$$

Следовательно, функция (4) обладает симметрией прямоугольного параллелограмма:

$$
W\left(-\xi_{1}, \xi_{2}, \delta_{1}, \delta_{2}\right)=W\left(\xi_{1},-\xi_{2}, \delta_{1}, \delta_{2}\right)=W\left(\xi_{1}, \xi_{2}, \delta_{1}, \delta_{2}\right),
$$

из которой вытекает [2] справедливость асимптотического представления

$$
W(\xi, \delta)=U(\xi, \delta)+o\left(|\xi|^{4}\right)+O\left(|\xi|^{4}\right) O(\delta),
$$

где $U(\xi, \delta)=V\left(\xi_{1} e_{1}+\xi_{2} e_{2}, \delta\right)$ - линейная ритцевская аппроксимация функционала $V$ по модам $e_{1}, e_{2}$. Таким образом, для ключевой функции имеет место асимптотическое представление

$$
W_{0}(\xi, \delta)=\frac{\lambda_{1}}{2} \xi_{1}^{2}+\frac{\lambda_{2}}{2} \xi_{2}^{2}+\frac{1}{4}\left(A \xi_{1}^{4}+2 B \xi_{1}^{2} \xi_{2}^{2}+C \xi_{2}^{4}\right)+o\left(|\xi|^{4}\right)+O\left(|\xi|^{4}\right) O(\delta),
$$

где

$$
\begin{gathered}
\lambda_{1}=\delta_{1}-\pi^{2} \delta_{2}, \quad \lambda_{2}=\delta_{1}-4 \pi^{2} \delta_{2}, \\
A=\int_{0}^{1} e_{1}^{4} d x=\frac{3}{2}, \quad B=\int_{0}^{1} e_{1}^{2} e_{2}^{2} d x=3, \quad C=\int_{0}^{1} e_{2}^{4} d x=\frac{3}{2} .
\end{gathered}
$$

Сократив на множитель $3 / 2$, получим функцию (5) с нормализованной главной частью:

$$
\widetilde{W}_{0}(\xi, \delta)=\widetilde{U}(\xi, \delta)+o\left(|\xi|^{4}\right)+O\left(|\xi|^{4}\right) O(\delta),
$$

где

$$
\widetilde{U}(\xi, \delta)=\frac{\widetilde{\lambda}_{1}}{2} \xi_{1}^{2}+\frac{\widetilde{\lambda}_{2}}{2} \xi_{2}^{2}+\frac{1}{4}\left(\xi_{1}^{4}+4 \xi_{1}^{2} \xi_{2}^{2}+\xi_{2}^{4}\right) .
$$

"Геометрический сюжет" бифуркации критических точек и первые асимптотики ветвей бифурцирующих точек (по закритическим приращениям управляющих параметров) для функции $\widetilde{W}_{0}(\xi, \delta)$ полностью определяются ее главной частью $\widetilde{U}(\xi, \delta)$, которая представляет собой "возмущенную двумерную сборку" (с коэффициентом двойного отношения $a=2$ ), четную по каждой переменной.

Критические точки определяются системой уравнений

$$
\begin{aligned}
& \frac{\partial \widetilde{U}}{\partial \xi_{1}}=\widetilde{\lambda}_{1} \xi_{1}+\xi_{1}^{3}+2 \xi_{1} \xi_{2}^{2}=0 \\
& \frac{\partial \widetilde{U}}{\partial \xi_{2}}=\widetilde{\lambda}_{2} \xi_{2}+2 \xi_{1}^{2} \xi_{2}+\xi_{2}^{3}=0
\end{aligned}
$$


все решения которой делятся на три типа:

1) 0-решение $\xi_{1}=\xi_{2}=0$;

2) 1-модовые решения $\xi_{1}= \pm \sqrt{-\widetilde{\lambda}_{1}}, \lambda_{1}<0, \xi_{2}=0$ и $\xi_{1}=0, \xi_{2}= \pm \sqrt{-\widetilde{\lambda}_{2}}, \lambda_{2}<0$;

3) 2 -модовые решения $\xi_{1}= \pm \sqrt{\left(\widetilde{\lambda}_{1}-2 \widetilde{\lambda}_{2}\right) / 3}, \xi_{2}= \pm \sqrt{\left(\widetilde{\lambda}_{2}-2 \widetilde{\lambda}_{1}\right) / 3}, \widetilde{\lambda}_{1}-2 \widetilde{\lambda}_{2}>0$, $\widetilde{\lambda}_{2}-2 \widetilde{\lambda}_{1}>0$.

Пусть $\theta_{1}=\widetilde{\lambda}_{1}-2 \widetilde{\lambda}_{2}, \theta_{2}=\widetilde{\lambda}_{2}-2 \widetilde{\lambda}_{1}$. Так как матрица Гессе функции $\widetilde{U}$ представима в виде

$$
\left(\begin{array}{cc}
\widetilde{\lambda}_{1}+3 \xi_{1}^{2}+2 \xi_{2}^{2} & 4 \xi_{1} \xi_{2} \\
4 \xi_{1} \xi_{2} & \widetilde{\lambda}_{2}+2 \xi_{1}^{2}+3 \xi_{2}^{2}
\end{array}\right)
$$

то, как нетрудно проверить, при $\theta_{1}>0$ и $\theta_{2}>0$ существуют четыре 2-модовые критические точки индекса 1 . Все 1-модовые точки при этом являются точками локальных минимумов, а нуль - критической точкой индекса 2. Рождение 1-модовых критических точек происходит при переходах параметров $\widetilde{\lambda}_{1}, \widetilde{\lambda}_{2}$ в области отрицательных значений.

Каустика (бифуркационная диаграмма функций [10]) $\Sigma_{\widetilde{U}}$ функции $\widetilde{U}$ разбивает плоскость управляющих параметров на шесть зон

$$
\begin{gathered}
\omega_{0}=\left\{\widetilde{\lambda}_{1}>0, \widetilde{\lambda}_{2}>0\right\}, \\
\omega_{1}=\left\{\widetilde{\lambda}_{1}<0, \widetilde{\lambda}_{2}>0\right\}, \\
\omega_{2}=\left\{\widetilde{\lambda}_{1}>0, \widetilde{\lambda}_{2}<0\right\}, \\
\omega_{3}=\left\{\widetilde{\lambda}_{1}<0, \widetilde{\lambda}_{2}<0, \theta_{1}<0, \theta_{2}>0\right\}, \\
\omega_{4}=\left\{\widetilde{\lambda}_{1}<0, \widetilde{\lambda}_{2}<0, \theta_{1}>0, \theta_{2}<0\right\}, \\
\omega_{5}=\left\{\widetilde{\lambda}_{1}<0, \widetilde{\lambda}_{2}<0, \theta_{1}>0, \theta_{2}>0\right\} .
\end{gathered}
$$

Каждой зоне соответствует свой расклад (bif-расклад) бифурцирующих критических точек: параметрам из зоны $\omega_{0}$ отвечает случай единственной критической точки (точка минимума в нуле), для $\omega_{1}, \omega_{2}$ - пара симметрично расположенных (относительно нуля) 1-модовых точек минимума и седло в нуле, для $\omega_{3}, \omega_{4}$ - пара симметрично расположенных 1-модовых точек минимума, пара 1-модовых седел и точка локального максимума в нуле, для $\omega_{5}$ - четверка симметрично расположенных 1-модовых точек минимума, четверка 2-модовых седел и точка локального максимума в нуле.

При обходе плоскости управляющих параметров против часовой стрелки вокруг нуля, начиная с зоны $\omega_{0}$, соответствующие метаморфозы линий уровней и распределений критических точек изображены на диаграмме (рис. 1).

В этом случае появляются те и только те расклады бифурцирующих критических точек (bif-расклады), которым соответствуют следующие целочисленные векторы: $(1,0,0),(2,1,0),(2,2,1),(4,4,1)^{1}$ (см. [1], [2]).

\footnotetext{
${ }^{1}$ Компонента $l_{k}$ строки $\left(l_{0}, l_{1}, l_{2}\right)$ равна количеству критических точек с индексом Морса $k$ в рассматриваемом раскладе бифурцирующих критических точек.
} 

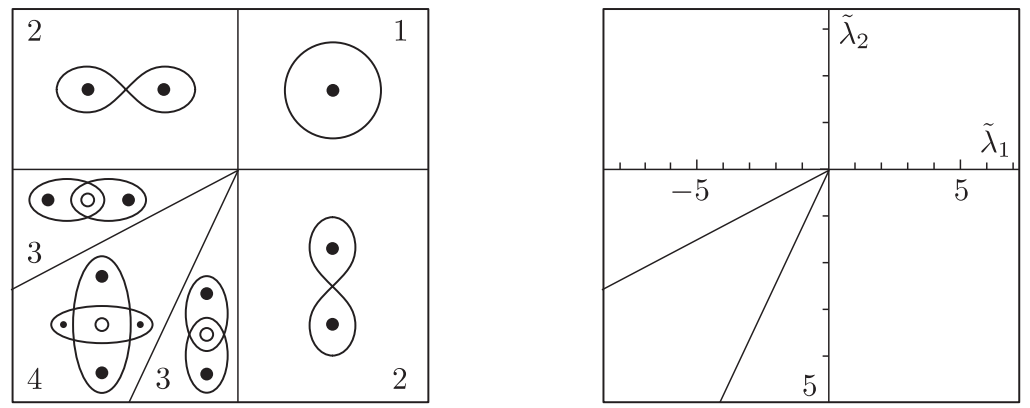

Рис. 1

Случай слабо неоднородной балки. Упругие равновесия слабо неоднородной балки описываются уравнением

$$
\frac{d^{2}}{d x^{2}}\left(q \frac{d^{2} w}{d x^{2}}\right)+\kappa \frac{d^{2} w}{d x^{2}}+\alpha w+w^{3}=0, \quad q(x)=1+\varepsilon \gamma(x),
$$

где $\varepsilon$ - малый параметр, $\gamma$ - гладкая функция. Уравнение (6) (также рассматриваемое на отрезке $[0,1]$ числовой оси при краевых условиях (2)) определяет экстремали функционала

$$
V=\int_{0}^{1}\left(\frac{1}{2}\left(q\left(\frac{d^{2} w}{d x^{2}}\right)^{2}-\kappa\left(\frac{d w}{d x}\right)^{2}+\alpha w^{2}\right)+\frac{w^{4}}{4}\right) d x .
$$

Наличие "весового" множителя $q$ не позволяет применять исследовательскую схему работы [1], так как из-за него не выполняется условие постоянства мод бифуркации. На нем основан вычислительный алгоритм [1]. Тем не менее, бифуркационный анализ решений краевой задачи (6), (2) также можно осуществить посредством редукции Ляпунова-Шмидта к ключевой функции более общего вида

$$
\widetilde{W}(\xi, \delta)=\inf _{w:\left\langle w, \widetilde{e}_{1}\right\rangle=\xi_{1},\left\langle w, \widetilde{e}_{2}\right\rangle=\xi_{2}} V\left(w, \alpha_{1}+\delta_{1}, \kappa_{1}+\delta_{2}\right),
$$

где $\left\{\widetilde{e}_{k}\right\}$ - "возмущенные" моды бифуркации

$$
\widetilde{e}_{k}=e_{k}+\varepsilon h_{k}+o(\varepsilon),
$$

образующие базис в 2-мерном корневом подпространстве оператора Гессе $\mathscr{H}=\mathscr{A}+\varepsilon \mathscr{B}$ в нуле (соответствующего функционалу (7)), где

$$
\begin{gathered}
\mathscr{A} u:=\frac{d^{4} w}{d x^{4}}+\kappa \frac{d^{2} w}{d x^{2}}+\alpha I, \quad \mathscr{B} w:=\frac{d^{2}}{d x^{2}}\left(\gamma \frac{d^{2} w}{d x^{2}}\right), \\
e_{k}=\sqrt{2} \sin (k \pi x)
\end{gathered}
$$

(элементы $\widetilde{e}_{k}$ не являются, вообще говоря, собственными функциями оператора $\mathscr{H}$ ).

Основная техническая трудность в построении главной части ключевой функции (8) состоит в вычислении коэффициентов $h_{k}$. Их можно определить на основе формулы ортогонального проектора на корневое подпространство возмущенного симметричного оператора, приведенной в монографии Маслова [3]. 
Итак, вместо собственных функций рассматриваем такие элементы $\widetilde{e}_{j}(\lambda), j=1,2$, для которых

$$
\frac{\partial f}{\partial x}(0, \lambda) \widetilde{e}_{j}(\lambda)=\sum_{k} \alpha_{j k}(\lambda) \widetilde{e}_{k}(\lambda) .
$$

Функции $\widetilde{e}_{j}(\lambda)$ будем называть корневыми.

Важным сопутствующим обстоятельством в предложенном здесь подходе является то, что входящие в эти соотношения функции $\alpha_{j k}(\lambda), \widetilde{e}_{j}(\lambda)$ будут гладко зависеть от $\lambda$. В качестве искомых базисных элементов можно взять

$$
\widetilde{e}_{k}(\lambda):=\mathbf{P}(\lambda)\left(e_{k}\right)
$$

где

$$
\mathbf{P}(\lambda)=\frac{1}{2 \pi i} \oint_{\ell} \mathscr{R}(\lambda, z) d z
$$

- ортопроектор на двумерное корневое подпространство, $\ell$ - окружность достаточно малого радиуса с центром в нуле (на комплексной плоскости), $\mathscr{R}(\lambda, z)$ - резольвента

$$
\mathscr{R}(\lambda, z)=(\mathscr{A}+\varepsilon \mathscr{B}-z I)^{-1} .
$$

Таким образом,

$$
\widetilde{e}_{k}=e_{k}+\varepsilon h_{k}+o(\varepsilon)
$$

где

$$
\begin{gathered}
h_{k}=\mathscr{M} e_{k}, \\
\mathscr{M}:=\frac{1}{2 \pi i} \oint_{\ell}(\mathscr{A}-z I)^{-1} \mathscr{B}(\mathscr{A}-z I)^{-1} d z .
\end{gathered}
$$

Итак, справедливо следующее утверждение.

ТеОрема 1. Возмущенные корневые векторы $\widetilde{e}_{k}, k=1,2$, можно представить в виде (9), где $h_{k}$ определяются соотношениями (10), (11).

Вычисление интегральных коэффициентов (по формуле (11)). Из предыдущих рассуждений следует, что для получения корневых векторов $\widetilde{e}_{k}$ необходимо вычислить интеграл

$$
\mathscr{M} e_{k}=\frac{1}{2 \pi i} \oint_{|z|=1}(\mathscr{A}-z I)^{-1} \mathscr{B}(\mathscr{A}-z I)^{-1} e_{k} d z
$$

с учетом краевых условий (2) (здесь $\ell$ - контур $|z|=1$ ).

Собственное число $z=0$ двукратно. Ему отвечают собственные элементы $e_{k}=$ $\sqrt{2} \sin (k \pi x), k=1,2$.

Заметим, что если $e_{k}$ - собственный вектор, отвечающий собственному числу $z_{k}$ : $\mathscr{A} e_{k}=z_{k} e_{k}$, то

$$
(\mathscr{A}-z I)^{-1}\left(\mathscr{A}-z_{k} I\right) e_{k}=0
$$

или

$$
(\mathscr{A}-z I)^{-1}\left(\mathscr{A}-z I+z I-z_{k} I\right) e_{k}=e_{k}-\left(z_{k}-z\right)(\mathscr{A}-z I)^{-1} e_{k}=0 .
$$


Отсюда получаем

$$
(\mathscr{A}-z I)^{-1} e_{k}=\frac{e_{k}}{z_{k}-z} .
$$

Таким образом, формулу (12) можно записать в виде

$$
\mathscr{M} e_{k}=\frac{1}{2 \pi i} \oint_{|z|=1} \frac{(\mathscr{A}-z I)^{-1} \mathscr{B} e_{k}}{z_{k}-z} d z .
$$

Очевидно, что оператор $\mathscr{A}$ с заданной областью определения и краевыми условиями является симметричным, а собственные функции в силу следствия теоремы 1 [1]; с. 91] образуют полную систему в $L^{2}[0,1]$.

Рассмотрим функцию $g(x)=\mathscr{B} e_{k}, g(x) \in L^{2}[0,1]$, и разложим ее в ряд по собственным функциям оператора $\mathscr{A}$ :

$$
g(x)=\sum_{n=1}^{\infty} c_{n} e_{n}(x)
$$

где

$$
c_{n}=\int_{0}^{1} g(s) e_{n}(s) d s=\int_{0}^{1} e_{n}(s)\left(\gamma(s) e_{k}^{\prime \prime}(s)\right)^{\prime \prime} d s .
$$

Интегрируя по частям, преобразуем интеграл (15) к виду

$$
c_{n}=-(n \pi)^{2} \int_{0}^{1} e_{n}(s) \gamma(s) e_{k}^{\prime \prime}(s) d s=\left(n k \pi^{2}\right)^{2} \int_{0}^{1} \gamma(s) e_{n}(s) e_{k}(s) d s .
$$

Пользуясь формулой (14) в интеграле (13), при $k=5 \pi^{2}, \alpha=4 \pi^{4}$ получаем следующие разложения:

$$
\begin{aligned}
\mathscr{M} e_{k}(x) & =\frac{1}{2 \pi i} \oint_{|z|=1} \frac{(\mathscr{A}-z I)^{-1}}{z} \sum_{n=1}^{\infty} c_{n} e_{n}(x) d z=\sum_{n=1}^{\infty} c_{n} \frac{1}{2 \pi i} \oint_{|z|=1} \frac{e_{n}(x) d z}{z\left(z_{n}-z\right)} \\
& =\sum_{n=3}^{\infty} c_{n} e_{n}(x) \frac{1}{2 \pi i} \oint_{|z|=1} \frac{1}{z_{n}}\left(\frac{1}{z}+\frac{1}{z_{n}-z}\right) d z=\sum_{n=3}^{\infty} \frac{c_{n} e_{n}(x)}{z_{n}}
\end{aligned}
$$

Здесь было учтено, что $z_{1}=z_{2}=0$ и

$$
\frac{1}{2 \pi i} \oint_{|z|=1} \frac{d z}{z^{2}}=0
$$

Таким образом, на основе (12) получаем для возмущенных собственных функций (в случае слабой неоднородности)

$$
\begin{aligned}
& M e_{1}=\sum_{n=3}^{\infty} \frac{2 \sqrt{2} n^{2}}{\left(n^{2}-4\right)\left(n^{2}-1\right)}\left(\int_{0}^{1} \gamma(s) \sin (n \pi s) \sin (\pi s) d s\right) \sin (n \pi x), \\
& M e_{2}=\sum_{n=3}^{\infty} \frac{8 \sqrt{2} n^{2}}{\left(n^{2}-4\right)\left(n^{2}-1\right)}\left(\int_{0}^{1} \gamma(s) \sin (n \pi s) \sin (2 \pi s) d s\right) \sin (n \pi x) .
\end{aligned}
$$


Теперь, когда известны первые асимптотики корневых векторов $\widetilde{e}_{1}, \widetilde{e}_{2}$, можно построить главную часть ключевой функции (с точностью до линейной замены координат)

$$
\begin{gathered}
W_{q}(\xi, \nu)=\frac{1}{2}\left(\nu_{1} \xi_{1}^{2}+\nu_{2} \xi_{2}^{2}+2 \nu_{3} \xi_{1} \xi_{2}\right)+\frac{1}{4}\left(A \xi_{1}^{4}+2 B \xi_{1}^{2} \xi_{2}^{2}+C \xi_{2}^{4}\right) \\
+o\left(|\xi|^{4}\right)+O\left(|\xi|^{4}\right) O(\nu, \varepsilon)+o(\nu, \varepsilon)
\end{gathered}
$$

где

$$
\begin{gathered}
\nu_{1}=\int_{0}^{1}\left(q\left(\frac{d^{2} \widetilde{e}_{1}}{d x^{2}}\right)^{2}-\left(\kappa_{1}+\delta_{1}\right)\left(\frac{d \widetilde{e}_{1}}{d x}\right)^{2}+\left(\alpha_{1}+\delta_{2}\right) \widetilde{e}_{1}^{2}\right) d x, \\
\nu_{2}=\int_{0}^{1}\left(q\left(\frac{d^{2} \widetilde{e}_{2}}{d x^{2}}\right)^{2}-\left(\kappa_{1}+\delta_{1}\right)\left(\frac{d \widetilde{e}_{2}}{d x}\right)^{2}+\left(\alpha_{1}+\delta_{2}\right) \widetilde{e}_{2}^{2}\right) d x, \\
\nu_{3}=\varepsilon \int_{0}^{1}\left(\gamma \frac{d^{2} \widetilde{e}_{1}}{d x^{2}} \cdot \frac{d^{2} \widetilde{e}_{2}}{d x^{2}}\right) d x, \quad B=\int_{0}^{1} e_{1}^{2} e_{2}^{2} d x=3 .
\end{gathered}
$$

После сокращения на множитель $3 / 2$ получаем искомое представление ключевой функции. Таким образом, справедливо следующее утверждение.

ТеОрема 2. Для ключевой функиии (8) имеет место представление

$$
\begin{gathered}
\widetilde{W}_{q}(\xi, \nu)=\frac{1}{2}\left(\widetilde{\nu}_{1} \xi_{1}^{2}+\widetilde{\nu}_{2} \xi_{2}^{2}+2 \widetilde{\nu}_{3} \xi_{1} \xi_{2}\right)+\frac{1}{4}\left(\xi_{1}^{4}+4 \xi_{1}^{2} \xi_{2}^{2}+\xi_{2}^{4}\right) \\
+o\left(|\xi|^{4}\right)+O\left(|\xi|^{4}\right) O(\widetilde{\nu}, \varepsilon)+o(\widetilde{\nu}, \varepsilon)
\end{gathered}
$$

где $\widetilde{\nu}_{j}$ определены соотношениями (10), (11), (16), (17)-(19).

По сравнению с функцией $W_{0}$, определенной равенством $(5)$, здесь появилось дополнительное слагаемое $\widetilde{\nu}_{3} \xi_{1} \xi_{2}$, разрушающее симметрию прямоугольника.

Исследование каустики. Так как в "неоднородном случае" появляется третий управляющий параметр $\widetilde{\nu}_{3}$, то дискриминантное множество будет двумерной поверхностью в трехмерном пространстве.

Рассмотрим систему уравнений, определяющую критические точки:

$$
\begin{aligned}
\frac{\partial \widetilde{U}}{\partial \xi_{1}} & =\widetilde{\nu}_{1} \xi_{1}+\xi_{1}^{3}+2 \xi_{1} \xi_{2}^{2}+\widetilde{\nu}_{3} \xi_{2}=0, \\
\frac{\partial \widetilde{U}}{\partial \xi_{2}} & =\widetilde{\nu}_{2} \xi_{2}+2 \xi_{1}^{2} \xi_{2}+\xi_{2}^{3}+\widetilde{\nu}_{3} \xi_{1}=0, \\
\operatorname{det} H & =\left|\begin{array}{cc}
\widetilde{\nu}_{1}+3 \xi_{1}^{2}+2 \xi_{2}^{2} & 4 \xi_{1} \xi_{2}+\widetilde{\nu}_{3} \\
4 \xi_{1} \xi_{2}+\widetilde{\nu}_{3} & \widetilde{\nu}_{2}+2 \xi_{1}^{2}+3 \xi_{2}^{2}
\end{array}\right|=0
\end{aligned}
$$

(здесь $H$ - матрица Гессе). 
Решая данную систему относительно $\widetilde{\nu}_{1}, \widetilde{\nu}_{2}, \widetilde{\nu}_{3}$ и переходя к полярным координатам $\xi_{1}=\rho \sin \varphi, \xi_{2}=\rho \cos \varphi$, получим

$$
\begin{aligned}
& \widetilde{\nu}_{1}=-\rho^{2} \frac{3+\cos (2 \varphi)^{3}}{2+\sin (2 \varphi)^{2}}=0, \\
& \widetilde{\nu}_{2}=-\rho^{2} \frac{3-\cos (2 \varphi)^{3}}{2+\sin (2 \varphi)^{2}}=0, \\
& \widetilde{\nu}_{3}=-3 \rho^{2} \frac{\sin (2 \varphi)^{3}}{4+2 \sin (2 \varphi)^{2}}=0 .
\end{aligned}
$$

На основе этой системы соотношений получено (в компьютерной среде Maple) следующее изображение каустики (рис. 2):

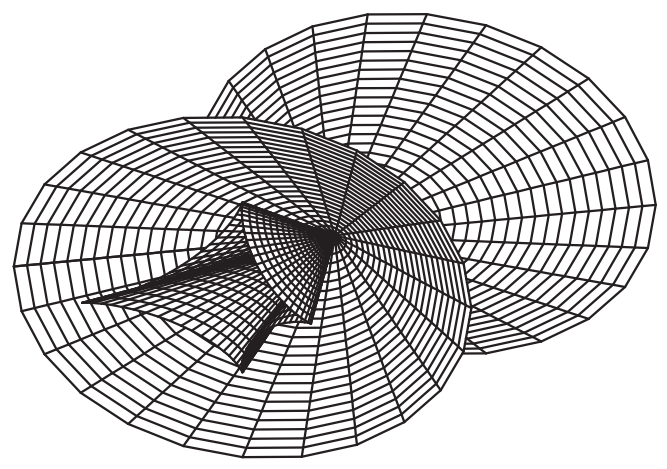

Рис. 2

Если зафиксировать $\widetilde{\nu}_{3}=$ const $\neq 0$, то получим двумерное сечение каустики, посредством которого удобно иллюстрировать появление метоморфоз линий уровней (в зависимости от изменения управляющих параметров); см. рис. 3.

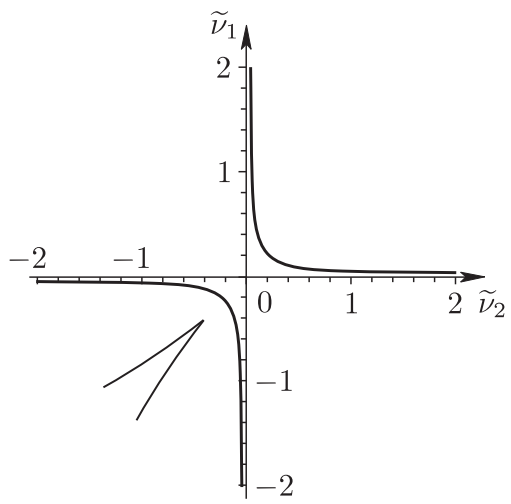

Рис. 3 

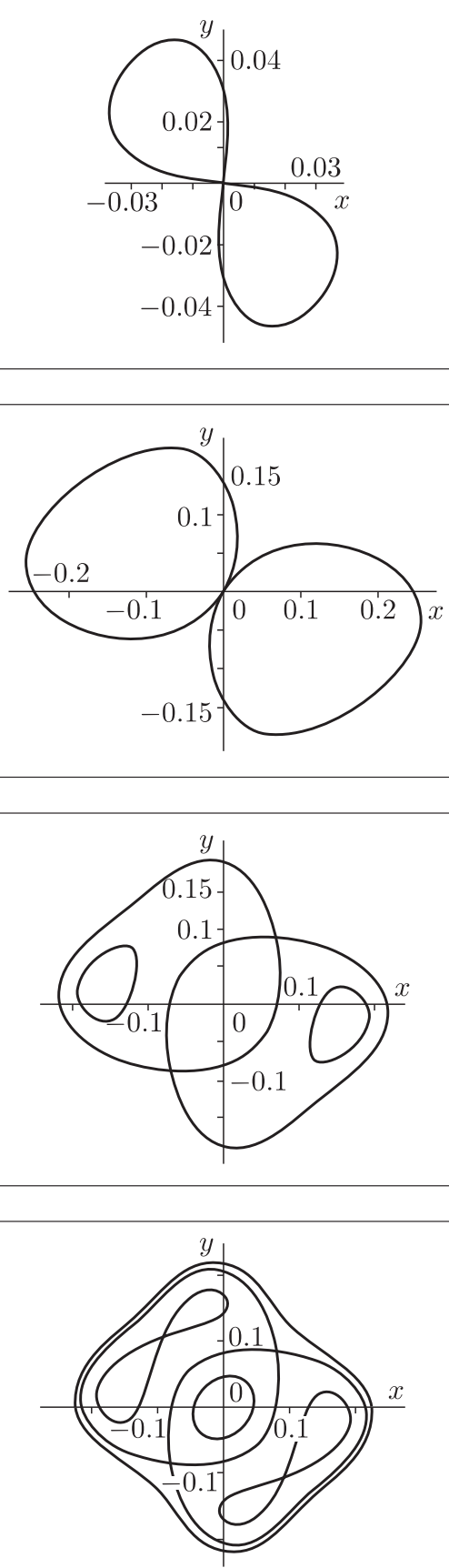
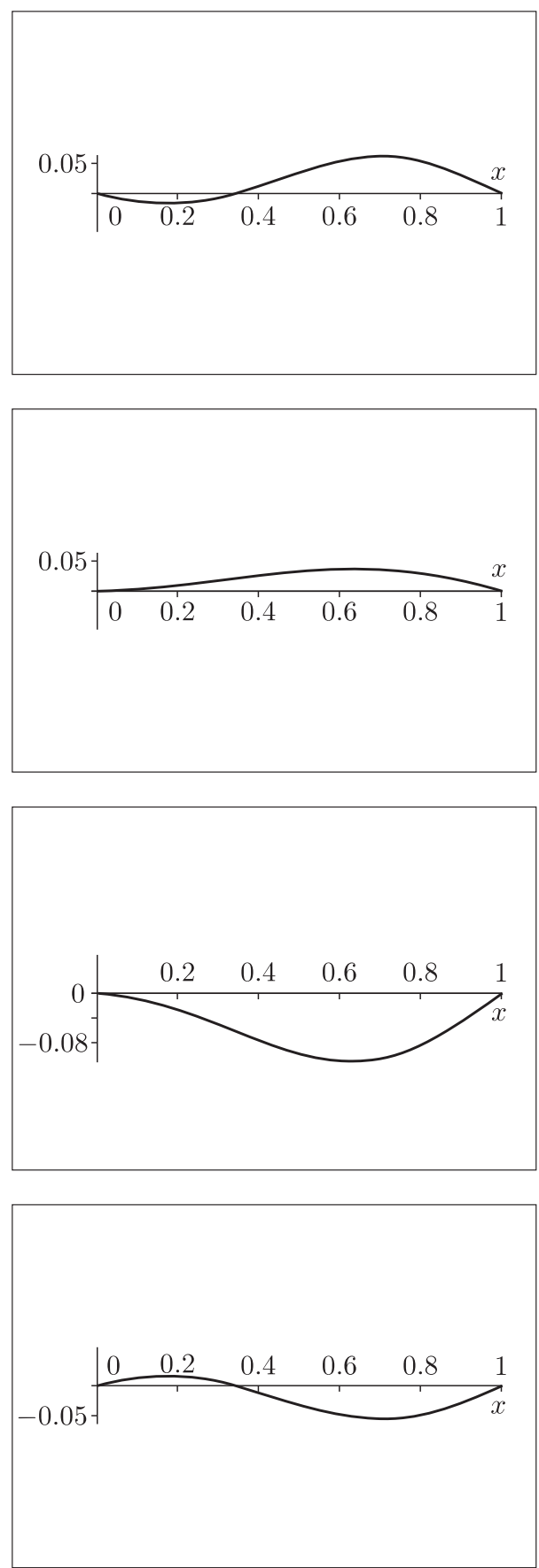

Рис. 4 
На рис. 4 изображены линии уровня главной части ключевой функции (при “типичных" значениях параметров $\widetilde{\nu}_{1}, \widetilde{\nu}_{2}, \widetilde{\nu}_{3}$ и функции $\left.\gamma=0,01 \cdot \sin (2 \pi x)\right)$ : сепаратрисные линии уровня ключевой функции и прогибы, соответствующие точкам минимума.

\section{СПИСОК ЦИТИРОВАННОЙ ЛИТЕРАТУРЫ}

[1] Б. М. Даринский, Ю.И. Сапронов, "О двухмодовых бифуркациях решений одной вариационной краевой задачи для уравнения четвертого порядка", Понтрягинские чтения, ХІ: Сб. трудов. Часть 1, ВГУ, Воронеж, 2000, 57-64.

[2] Б. М. Даринский, Ю. И. Сапронов, С. Л. Царев, "Бифуркации экстремалей фредгольмовых функционалов", Соврем. проблемъ матем. Фундам. напр., 12 (2004), 3-140.

[3] В. П. Маслов, Асимптотические методы и теория возмущений, Наука, М., 1988.

[4] Д. В. Костин, "Ортопроектор теории возмущения линейных операторов и бифуркации равновесий слабо неоднородной упругой балки", Труды Воронежской зимней математической школы С.Г. Крейна, ВГУ, Воронеж, 2006, 106-113.

[5] Ю. О. Мітропольский, Б. І. Мосеенков, Дослідження коливань в системах з розподіленими параметрами (асимптотичні методи), Вид-во Київського ун-ту, Киёв, 1961.

[6] J. M. T. Thompson, H. B. Stewart, Nonlinear Dynamiks and Chaos, Geometrical methods for engineers and scientists, John Wiley \& Sons, Chichester, 1986.

[7] Б. С. Бардин, С. Д. Фурта, "Локальная теория существования периодических волновых движений бесконечной балки на нелинейно упругом основании", Актуальные проблемы классической и небесной механики, Эльф, М., 1998, 13-22.

[8] Ю.А. Изюмов, В.И. Сыромятников, Фазовые переходы и симметрия кристаллов, Наука, М., 1984.

[9] М. А. Красносельский, Н. А. Бобылев, Э. М. Мухамадиев, "Об одной схеме исследования вырожденных экстремалей функционалов классического вариационного исчисления", Докл. АН СССР, 240:3 (1978), 530-533.

[10] В.И. Арнольд, А.Н. Варченко, С. М. Гусейн-Заде, Особенности дифферениируемых отображений. Классификачия критических точек каустик и волновых фронтов, Наука, М., 1982.

[11] М. А. Наймарк, Линейнье дифференииальные операторы, Наука, М., 1969.

Д. В. Костин

Воронежский государственный университет

E-mail: dvkostin@rambler.ru
Поступило 18.01.2007 